\title{
Correlación entre hemograma y frotis sanguíneo para determinar $E$. canis en la vereda Peñitas de Puente Nacional
}

\section{Correlation between blood count and blood smear to determine $E$. canis in the Peñitas path of Puente Nacional}

\author{
Adriana Milena González G. ${ }^{1}$, Eduar Fernando Rojas H. ${ }^{1}$, Martín Orlando \\ Pulido- Medellín², Diego José García- Corredor ${ }^{3}$
}

\section{Resumen}

Estudio serológico de Ehrlichia canis en la vereda Peñitas, del municipio de Puente Nacional, Santander, mediante la correlación de frotis sanguíneo y cuadro hemático. Se recolectaron 80 muestras de sangre en caninos para el cuadro hemático y el frotis sanguíneo, buscando encontrar en este último mórulas de E. canis, y en el hemograma, los cambios a nivel sanguíneo en aquellos individuos que resultaran positivos. Se realizó un análisis descriptivo tabulando la información con datos positivos y negativos obtenidos de cada animal; los resultados se interpretaron de acuerdo con las variables: raza, edad, sexo, antecedentes clínicos, estado general, planes de vermifugación y las variaciones del cuadro hemático. Para determinar la relación entre los casos positivos y cada una de las variables se utilizó la prueba de Probabilidad de Fisher. Las pruebas positivas arrojaron un resultado de $26,25 \%$ de presencia de E. Canis. No se encontraron

\begin{abstract}
It is a serological survey of Ehrlichia canis in the Peñitas path of Puente Nacional municipality, Santander, through the correlation of blood smear and blood count. Were collected 80 samples of canine blood for blood count and blood smear to find in the last one E. canis morulae and in blood analysis changes in blood level in those positive individuals. Was performed a descriptive analysis tabulating the information with positive and negative data obtained from each animal; the results were interpreted according to the variables: breed, age, sex, medical history, general state, dewormed plans and changes in blood count. To determine the relation between the positive cases and each of the variables was used the Fisher probability test. The positive tests result of $26.25 \%$ for $E$. Canis. There were no significant differences in prevalence associated with variables of the blood count. It shows presence of $E$. canis in Peñitas path. The obtained results confirm the
\end{abstract}

Médico Veterinario Zootecnista. Universidad Pedagógica y Tecnológica de Colombia. Tunja. e-mail: admigogo@hotmail.com; fhermj1@hotmail.com

2 Médico Veterinario. Esp. Docente Escuela de Medicina Veterinaria y Zootecnia. Universidad Pedagógica y Tecnológica de Colombia. Tunja. e-mail: mopm1@hotmail.com

3 Médico Veterinario Zootecnista. Joven Investigador Colciencias-Universidad Pedagógica y Tecnológica de Colombia. Tunja. e-mail: pibegarcia@yahoo.es 
diferencias significativas de prevalencia asociadas a las variables de los resultados del hemograma. Se demuestra la presencia de E. canis en la vereda Peñitas. Se confirma la importancia de implementar un programa de control y prevención de la diseminación del agente causal, mejorando los planes de vermifugación de los caninos.

Palabras clave: Ehrlichia canis, Caninos,

Ricketsia. (Fuente: DeCS) importance of implementing a program to control and prevent the spread of causal agent, improving dewormed plans in canines.

Key words: Ehrlichia canis, Canine, Ricketsia. (Source: DeCS)

18 - Adriana Milena González G, Eduar Fernando Rojas H, Martín Orlando Pulido-Medellín, Diego José García-Corredor 


\section{Introducción}

Las garrapatas y las enfermedades que transmiten son, sin duda, un tema de gran interés en la práctica diaria de la Clínica de Animales de Compañía, de todo el mundo. La migración cada vez más generalizada de personas y los medios de transporte más eficientes han derribado las fronteras entre los países, generando un intercambio cada vez más activo de parásitos y enfermedades alrededor del mundo. Las garrapatas son importantes porque todas son parásitas y porque transmiten gran cantidad de enfermedades al humano y a los animales domésticos; en esto, sólo son superadas por los mosquitos. Las garrapatas albergan y transmiten al humano y a los animales protozoarios, virus, bacterias, rickettsias y toxinas.

El agente etiológico de la erlichiosis monocítica canina (EMC), la ricketsia Ehrlichia canis (E. canis), es una bacteria Gram negativa, cocoide pleomórfica pequeña, que parasita el citoplasma de los monocitos circulantes, en grupos de organismos denominados mórulas. La enfermedad es también conocida como ricketsiosis canina, fiebre hemorrágica canina, enfermedad del perro rastreador, tifus de la garrapata canina, desorden hemorrágico de Nairobi y pancitopenia tropical canina, nombres que representan diferentes aspectos de una misma enfermedad. Esta enfermedad es reconocida como una patología infecciosa importante y potencialmente fatal de los perros y otros miembros de la familia Canidae. E. canis fue identificada por primera vez en Algeria, en 1935. Históricamente, la enfermedad cobró mucha importancia durante la Guerra de Vietnam, causando la muerte de cientos de perros militares. Posteriormente se le prestó atención en 1987, cuando E. chaffeensis, un organismo muy emparentado, fue identificado como la causa de la erlichiosis monocítica humana. Subsecuentemente, en 1996, se demostró que $E$. chaffeensis causa signos de enfermedad en los perros indistinguible de la infección provocada por E. canis.

Son pocos los estudios de prevalencia de $E$. canis realizados en Colombia; aunque se ha diagnosticado en el medio, se desconoce la presencia como tal del agente causante en la zona de estudio. La enfermedad ha sido reportada en Manizales (Benavides y Ramírez, 2003), en Villavicencio (Parrado et al., 2003), en Medellín (Gómez et al., 2006) y, recientemente, en Cali
(Silva et al., 2008). En Montería se hizo un estudio de prevalencia (Espitia et al., 2000), que no encontró presencia de enfermedad en la zona.

Las condiciones medioambientales del municipio de Puente Nacional, Santander, favorecen la presencia de parásitos como las garrapatas, que desempeñan un papel importante en la transmisión de numerosos agentes patógenos, entre ellos Ehrlichia canis, capaz de producir alteraciones severas en la salud de los caninos; además, la constante exposición de éstos al medioambiente y la falta de aplicación periódica de tratamientos preventivos, con antiparasitarios externos a los perros de finca, facilita la infestación con dicho parásito.

En la actualidad, resulta difícil dar un diagnóstico confirmativo de la enfermedad en campo, basado solamente en la apreciación de los signos clínicos del paciente, debido a que son inespecíficos (fiebre, anorexia, depresión, pérdida de peso, epistaxis, hemesis) y a la variación presentada de acuerdo con la fase de la enfermedad, lo que también hace probable que tales signos se confundan con otras patologías y, por consiguiente, no se llegue a un tratamiento adecuado.

Por lo anterior, fue de vital importancia realizar este estudio, en el cual se determinó la presencia de $E$. canis en perros de la vereda Peñitas del municipio de Puente Nacional, Santander, correlacionando el cuadro hemático con el frotis sanguíneo, lo que puede servir de referencia a la hora de diagnosticar enfermedades en perros con signos y síntomas compatibles con Ehrlichiosis canina en la zona, además de establecer el riesgo al que puede estar sometida la población canina del área.

\section{Materiales y Métodos}

El estudio se realizó en la vereda Peñitas del municipio de Puente Nacional; se eligió esta vereda por la presencia de caninos de finca expuestos a garrapatas, en su mayoría de raza criolla y con escaso control antiparasitario, y por las facilidades que brindaron los propietarios para tomar las muestras y recolectar los datos requeridos para el estudio; además, por la cercanía de la vereda al casco urbano.

El municipio de Puente Nacional tiene una extensión de 25.320,6 ha, y la vereda Peñitas, 668,7 ha. Según el censo de vacunación antirrábica canina (Hospital Integrado San Antonio, 2008), 
en el municipio existe una población de 2.500 caninos, en la vereda, una población de 120 caninos.

Para el estudio se tomaron al azar 80 caninos de diferentes fincas de la vereda, en la mayoría de raza criolla, que tuvieron contacto previo con garrapatas; de ellos los dueños suministraron datos como edad, antecedentes clínicos, estado físico general y presencia o ausencia de planes de vermifugación.

Método de recolección y envío de muestras. En el momento de la toma de las muestras en las fincas, se realizó adecuada sujeción del perro con bozal. Se procedió a ocluir la vena con presión digital o torniquete, luego se introdujo el vacutainer de calibre 21 G x 1 1/2 y se recogió la sangre en un tubo tapa lila (con anticoagulante); se tomaron $5 \mathrm{ml}$ de sangre por canino, por tubo. Luego los tubos se almacenaron en una cava de icopor y en refrigeración con el fin de mantener las muestras en condiciones ideales; esta toma de muestras se realizó de tal forma que el tiempo de transporte de las muestras al laboratorio fuese el permitido y con el almacenamiento adecuado. Cada muestra se rotuló con información como el nombre del canino, raza, sexo, edad y planes de vermifugación previos.

Se procesaron las muestras para cuadro hemático y frotis sanguíneo en el Laboratorio Clínico Veterinario de la Clínica de Grandes y Pequeños Animales, de la Uptc.

Diseño experimental. Para realizar este estudio se muestrearon caninos de diferentes edades y en diferentes fincas. Para obtener un análisis adecuado de los resultados y de la información obtenida se aplicó un método estadístico adecuado que permitió trabajar una serie de valores medibles respecto a la población de estudio, y así lograr concluir y alcanzar el objetivo de la investigación; dichos resultados se relacionaron con otros aspectos por estudiar, como las variables, así como los resultados del cuadro hemático de cada animal y el resultado del frotis sanguíneo (Ross, 2000; Charles, 2006).

Por medio del procedimiento estadístico "Determinación de tamaño de muestra para poblaciones finitas" se estableció la cantidad de animales por muestrear, teniendo en cuenta que la población de caninos de la vereda Peñitas es de
120, según el censo de vacunación antirrábica del 2009. Luego de aplicar el método estadístico para este estudio, se tomó una población de 80 animales considerados como muestra representativa para determinar presencia de Ehrlichia canis en la vereda Peñitas, con un intervalo de confianza del $91,5 \%(0,9150)$, como se muestra a continuación: El intervalo de confianza del $91,5 \%$ (0.9150) se da con un alfa del 8,5\% $(0,0850)$, para obtener un tamaño de muestra de 79 individuos como mínimo para considerar una muestra representativa; en este caso la población de 80 caninos es suficiente para indicar una prevalencia en el lugar de estudio.

De esta forma se llevó a cabo el Método Aleatorio Simple de Corte Transversal de 80 caninos que se seleccionaron por el método aleatorio simple del censo de vacunación antirrábica de 2009 de la vereda Peñitas; en dicha selección se tomó en cuenta como fuente de información complementaria la edad, la raza, el sexo, el estado físico general, los antecedentes clínicos y los planes de vermifugación previos, entre otros aspectos, como los resultados del frotis sanguíneo y los obtenidos en el cuadro hemático canino. De esta manera se determinó la presencia de Ehrliquia canis en la vereda Peñitas del municipio de Puente Nacional, Santander (Ross, 2000; Charles, 2006).

En el análisis estadístico se tomó como parte fundamental el cálculo en porcentaje de la presencia de las variables planteadas, obteniendo así tablas y gráficos. Para facilitar el análisis, comprensión y, finalmente, concluir el estudio, se hizo una correlación con los resultados de los cuadros hemáticos y los datos suministrados por los propietarios, y finalmente se realizaron algunas recomendaciones.

Análisis estadístico: Se aplicó la Prueba de Probabilidad de Fisher para establecer si hay asociación o independencia entre la presenciaausencia de $E$. canis con las diferentes variables. La prueba se utiliza para analizar datos discretos (nominales -en este caso- u ordinales); se usa cuando los puntajes de dos muestras seleccionadas independientemente al azar pertenecen respectivamente a clases mutuamente excluyentes, es decir, cada individuo obtiene uno de los dos posibles puntajes; los puntajes se presentan mediante frecuencias en una tabla de contingencia de $2 \times 2$; cada variable tiene dos clases mutuamente excluyentes. 


\section{Resultados y Discusión}

Como resultado de este estudio se obtuvo una prevalencia de $26,25 \%$ de casos positivos, y $73,75 \%$ de casos negativos. Cifras distantes a las de un estudio realizado en la ciudad de Cali, donde de 101 pruebas de ELISA realizadas en sangre de caninos para análisis diagnóstico específico de $E$. canis, el $49,5 \%$ resultaron positivas, y el 50,5\%, negativas (Silva et al., 2008); el mayor porcentaje de casos positivos se debe a la especificidad de la prueba ELISA en la confirmación de E. canis; de los casos positivos se encontró que por raza se presenta más en criolla $(22,5 \%)$, y es menor en Pastor ovejero, Pincher y Poddle (cada una 1,25\%), y seronegativas en Labrador y Cocker; sin embargo, hay que señalar que estos resultados se dan debido a que la mayoría de caninos muestreados eran de raza criolla (75 animales), y sólo había un animal de cada una de las otras razas, por lo cual el porcentaje para estas últimas no fue muy importante, comparado con resultados obtenidos en otros estudios, en donde la raza labrador presentó positividad del 7,9\%; Podle, del 7\%; Cocker y Criollo (mestizo), del 2,97\% (Silva et al., 2008).

Del $26,25 \%$ de casos positivos, un $16,25 \%$ eran machos y un 10\%, hembras; estos resultados muestran una elevación en la frecuencia de presentación en animales machos respecto a las hembras, lo que ya observó Burke y Cunha (2004), quienes reportan que los machos son más afectados, en proporción 4:1, que las hembras, sin que exista una explicación clara de la causa; resultado que coincide con Ortegón et al. (2004) y Ettinger (1997), quienes encontraron predisposición de los hemoparásitos por canes machos.

A pesar de que no hay relación entre la edad de los animales y la presentación de la enfermedad (Sainz et al., 2000), en este estudio se encontró que se presentaron animales positivos en todos los grupos etarios; sin embargo, el grupo más afectado fue el de los animales de edad adulta (3 y 5 años), debido, posiblemente, a que la mayoría de los caninos muestreados están en este rango de edad.

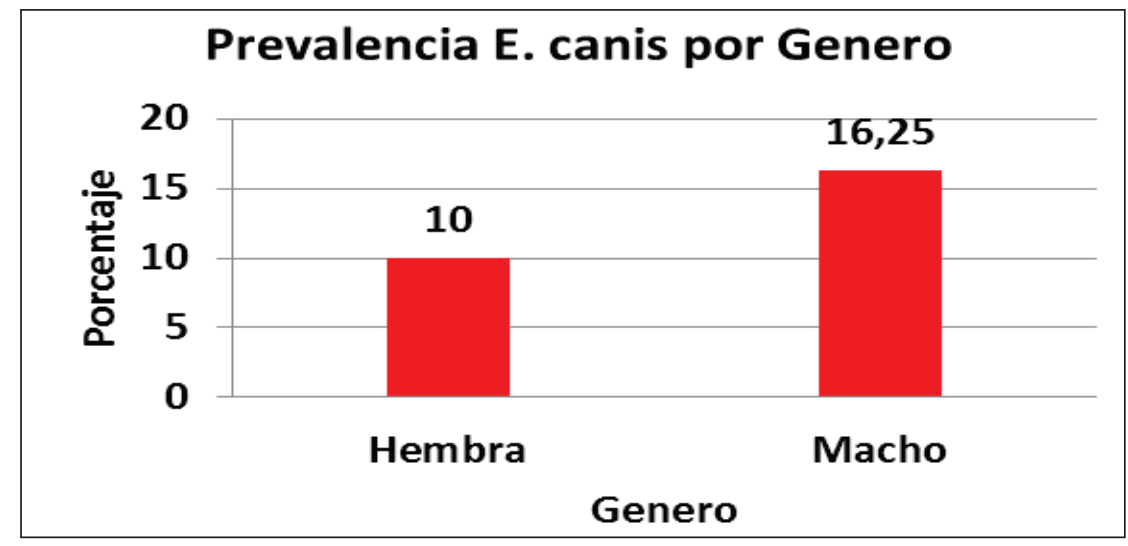

Figura 1. Prevalencia de $E$. canis por género

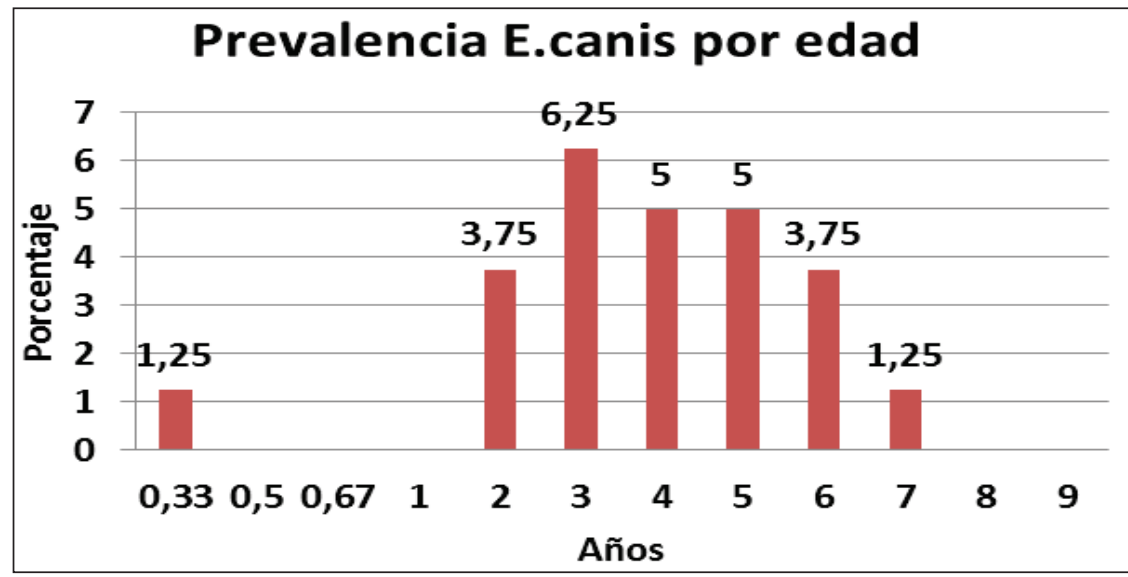

Figura 2. Prevalencia de E. canis por edad 
Se encontró más prevalente en individuos sin antecedentes clínicos (25\%), que en los que sí tenían $(1,25 \%)$, y más en individuos de estado físico general bueno $(22,5 \%)$ que en regular $(3,75 \%)$; esto puede basarse en la fase de la enfermedad en la que se encontraban los caninos en el momento del muestreo: en la fase aguda los signos clínicos pueden ser leves y no específicos, aunque en algunos casos pueden ser severos y comprometer la vida (Waner et al., 2000).

Parrado et al. (2003) encontraron que en los animales problema los antecedentes fueron muy variados, entre ellos: decaimiento, presentado en el $75 \%$; membranas mucosas pálidas, en el $67,86 \%$; lifadenitis, en el $67,86 \%$, y pérdida de peso, en el $64.28 \%$ de los animales. La variación de los signos clínicos puede deberse a la condición corporal, a la capacidad de respuesta inmunológica y a la fase de la enfermedad que presentaban los animales al momento de ser examinados.

Al indagar a los propietarios acerca de la última vez que vermifugaron a los perros, la mayoría respondió hace más de tres meses, por lo cual se encontró más prevalente en estos individuos $(25 \%)$ que en los vermifugados cada 3 meses $(1,25 \%)$, (Gráfico 7). El uso continuo de garrapaticidas, comúnmente disponibles para el control de las infestaciones por garrapatas, es altamente recomendable (Ristic y Holland, 1992).

Las alteraciones hematológicas (trombocitopenia, leucopenia y anemia) son frecuentes en los casos de ehrlichiosis canina (Ettinger, 1992). La trombocitopenia es el hallazgo hematológico más común y consistente en la Ehrlichiosis Monocitica Canina (EMC) aguda. Un aumento concurrente y significativo del volumen medio de plaquetas es también usualmente visto, reflejando una trombopoyesis activa. En la fase aguda de la enfermedad es común la leucopenia y la anemia moderada (usualmente normocítica, normocrómica, no regenerativa). La trombocitopenia moderada es un hallazgo común en la fase subclínica de la enfermedad. Puede haber un descenso en el número de los neutrófilos. Los parámetros eritrocíticos no son afectados normalmente en esta etapa de la enfermedad. La trombocitopenia severa, leucopenia y anemia se presentan más comúnmente durante la fase crónica de la EMC. La pancitopenia severa es la característica de la fase crónica grave, y ocurre como resultado de una médula ósea hipocelular suprimida (Waner et al., 2000).
Según Greene (2000), las alteraciones hematológicas se comprueban mejor en infecciones por $E$. canis e incluyen anemia $(82 \%)$, que suele ser no regenerativa; trombocitopenia $(82 \%)$, y leucopenia $(32 \%$, de la cual el $20 \%$ tuvo neutropenia). La pancitopenia suele resultar de hipoplasia de todas las células precursoras de la médula ósea y ocurre en fase crónica grave ( $18 \%$ de los casos). Un dato que se ha publicado constantemente es trombocitopenia en todas las etapas de ehrlichiosis por E. canis; sin embargo, debido a que con frecuencia es una prueba de selección para ehrlichiosis, es posible que se haya estimado en exceso esta proporción.

Para este estudio, la prevalencia de E. canis, según las variables del hemograma, indica que se puede presentar en cualquier concentración de hematocritos, hemoglobina, eritrocitos, reticulocitos, plaquetas y leucocitos; lo que indica que los resultados del cuadro hemático para la correlación con el frotis sanguíneo no fueron significativos, es decir, no coincidieron con parte de la revisión bibliográfica y los resultados de algunos estudios en los que el grado de concordancia encontrado entre el examen hematológico y la técnica directa de ELISA (84.7 \pm $11.0 \%$ ) es considerado como muy bueno (Hoyos et al, 2007).

Pero este estudio sí se relaciona con los resultados obtenidos en el estudio realizado en Villavicencio, en donde las variables del cuadro hemático (hematocrito, hemoglobina, recuento de leucocitos y plaquetas) comparada entre los pacientes negativos a frotis sanguíneo, pero positivos a serología, no fueron significativos $(p>0.05)$, indicando que los resultados del hemograma no orientan el diagnóstico; esto se debió a una gran variación en los datos, por lo que se hace necesaria la implementación de otras pruebas diagnósticas específicas que nos permitan la aproximación a un diagnóstico confirmativo de ehrlichiosis (Parrado et al., 2003).

Otros datos arrojados por este estudio y que no se relacionan directamente con la presencia de $E$. canis fueron: Es más probable establecer o identificar la presencia de $E$. canis en individuos que no hayan presentado antecedentes clínicos (positivos 20/21 $=95 \%$ ), con reticulocitos en concentraciones normales $(16 / 21=76 \%$ o con policromatofilia ligera $(17 / 21=81 \%)$, comprobado por la prueba de Fisher al indicar una asociación entre estas variables y la presencia-ausencia del agente. 


\section{Literatura citada}

Benavides, V. A., Ramírez, G. F. (2003). Caso Clínico de Ehrlichiosis Canina. Revista Colombiana de Ciencias Pecuarias 16(3), 268274.

Burke, A., Cunha, M. D. (2004). Chief, Infections Disease Division, inthrop University Hospital. Professor of Medicine, State University of New York Stony Brook School of Medicine. URL disponible en: http//www.emedicine. com/cgibin/foxweb.exe/screened./em/ga? . [Fecha de acceso 13 de marzo de 2012].

Charles, W. L., Josephf, H. C., McDaniel, D. (2006). Marketing. Edition: 8. Publicado por Cengage Learning Editores. Recuperado de: http:// www.revistaciencias.com/publicaciones/ EEZZpAIVFpLeJclctD.php

Ettinger, S. (1997). Tratado de Medicina Interna Veterinaria. Cuarta edición, volumen 1. Buenos Aires: Intermedia.

Greene, C. (2000). Enfermedades infecciosas de perros y gatos. Segunda edición. Philadelfia. México D. F. 404-414.

Hospital Integrado San Antonio, Puente NacionalSantander. Área de Saneamiento Ambiental (2008). Censo de vacunación antirrábica canina y felina.

Hoyos, L., Li, O., Alvarado, A., Suárez, F., Díaz, D. (2007). Evaluación del examen hematológico en el diagnóstico de ehrlichiosis canina. Revista Investigaciones Veterinarias Perú. Facultad de Medicina Veterinaria, Universidad Nacional Mayor de San Marcos, 18 (2), 129135.
Ortegón, S. E., Molina, G. J., Álvarez, P. L. (2004). Estudio retrospectivo de la prevalencia de hemoparásitos en caninos del distrito de Cartagena, Colombia.

Parrado, M., Vargas, F., Hernández, G., Vergara, H. (2003). Asociación de los resultados de una prueba serológica (ELISA) y frotis sanguíneo en caninos con sintomatología compatible de ehrlichiosis. Revista Orinoquía, 7(1-2), 6-11.

Ristic, M., Holland, C. (1992). Ehrlichiosis canina. En: Manual de las enfermedades infecciosas en pequeños animales, 169-186. Buenos Aires: Editorial Médica Panamericana.

Ross, S. (2000). Probabilidad y estadística para ingenieros. Segunda edición. Sevilla, España: McGraw Hill.

Sainz,A. (2000). Aspectos clínicosyepizootológicos de la ehrlichiosis canina. Estudio comparado de la eficacia terapéutica de la doxiciclina y dipropionato de imidocarb. Tesis Doctoral. Universidad Complutense de Madrid, España.

Silva, M. R., Sánchez, U. N., Loaiza, E. A. (2008). Reporte de presentación de Ehrlichia canis en muestras sanguíneas de caninos en la ciudad de Cali, Colombia. Revista de la Universidad de Caldas, 24(3), 39-43. Manizales, Colombia.

Waner, T., Leykin, I., Shinitsky, M., Sharabani, E., Buch, H., Keysary, A. (2000). Detection of platelet-bound antibodies in beagle dogs after artificial infection with Ehrlichia canis. Vet Immunol Immunopathol, 77, 145-150.

Fecha de Recepción: 15 de abril de 2012 Fecha de Aceptación: 17 de abril de 2013 